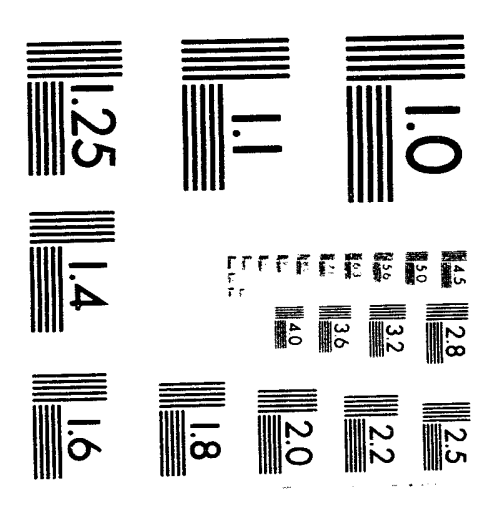



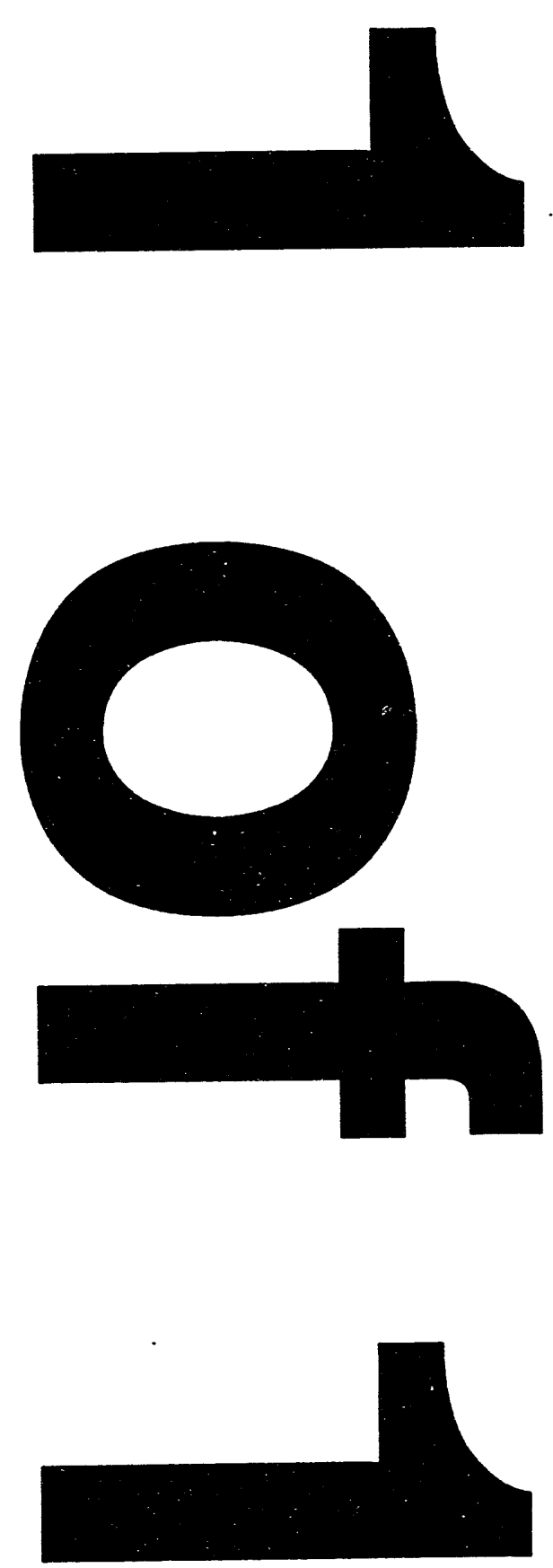


\section{LA-UR $94-713$}

TITLE: ANTIPROTON PRODUCTION IN HEAVY ION COLLISIONS

AUTHOR(S): Barbara ${ }^{T}$. Jacak, P-2

SUBMITTED TO: Presented at NATO Advanced Study Institute on "Hot and Dense Nuclear Matter" in Bodrum, Turkey, October 5, 1993

\section{DISCLAIMER}

This report was prepared as an account of work sponsored by an agency of the United States Government. Neither the United States Government nor any agency thereof, nor any of their employees, makes any warranty, express or implied, or assumes any legal liability or responsibility for the accuracy, completeness, or usefulness of any information, apparatus, product, or process disclosed, or represents that its use would not infringe privately owned rights. Reference herein to any specific commercial product, process, or service by trade name, trademark, manufacturer, or otherwise does not necessarily constitute or imply its endorsement, recommendation, or favoring by the United States Government or any agency thereof. The views and opinions of authors expressed herein do not necessarily state or reflect those of the United States Government or any agency thereof.
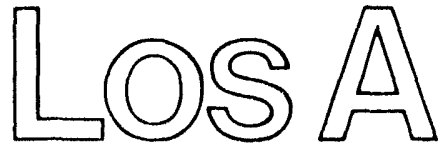
ST NO $26295 / 81$
MASTER

Los Alamos National Laboratory Los Alamos,New Mexico 87545 do

U/STIBBUTION OF THIS DOCUMENT IS UNLIMITES 


\title{
ANTIPROTON PRODUCTION IN HEAVY ION COLLISIONS
}

\author{
Barbara V. Jacak \\ for the NA44 Collaboration \\ Los Alamos National Laboratory \\ Los Alamos, New Mexico 87545 \\ USA
}

\section{INTRODUCTION}

In high energy $\mathrm{p}-\mathrm{p}$ and $\alpha-\alpha$ collisions, baryons are observed predominantly at rapidities near those of target and projectile; the mean rapidity shift of projectile and target nucleons is approximately one unit. ${ }^{1}$ In the central rapidity region, the number of baryons is quite small. In fact, the number of baryons and antibaryons is rather similar, indicating that most of these baryons are CREATED particles rather than projectile and target fragments. At the ISR, $\bar{p} / p \approx 0.4-0 . .^{2}$

Antibaryon production is of interest in heavy ion collisions as enhanced antiquark (and consequently, antibaryon) production has been predicted as a potential signature of quark-gluon plasma formation. ${ }^{3}$ Antibaryons also provide a sensitive probe of the hadronic environment, via annihilation ${ }^{4}$ and/or mean field effects upon their final distributions ${ }^{5}$. However, the collision dynamics also affect the baryon and antibaryon distributions. Baryons are more shifted toward midrapidity in nucleus-nucleus and pnucleus collisions than in p-p collisions, ${ }^{6,7}$, increasing the probability of annihilating the antibaryons. ${ }^{4}$ The interpretation of antibaryon yields is further complicated by collective processes which may take place in the dense hadronic medium formed in nucleus-nucleus collisions. Jahns and coworkers have shown that multistep processes can increase antibaryon production near threshold. ${ }^{8}$ At SPS energies, a mechanism allowing fusion of color strings stretched between individual colliding nucleons into color ropes with higher potential has been shown to enhance antibaryon production. ${ }^{9}$ To further complicate matters, annihilation of antiprotons by protons may be screened when the hadron density is very high, as suggested by Kahana and coworkers. ${ }^{10}$

Antiproton production is clearly very interesting, but is sensitive to a combination 
of processes taking place in the collision. The final number of observed antiprotons depends on the balance between mechanisms for extra antiproton production beyond those from the individual nucleon-nucleon collisions and annihilation with surrounding baryons. We can hope to sort out these things by systematic studies, varying the system size and beam energy. I will review what is known about antiproton production at both the AGS and SPS, and look at trends going from p-p to p-nucleus to nucleus-nucleus collsions.

\section{AGS RESULTS}

Four experiments at the AGS have measured antiproton distributions in nucleusnucleus collisions at $14.6 \mathrm{GeV} /$ nucleon: $\mathrm{E} 802 / \mathrm{E} 859^{11}, \mathrm{E} 814^{12}, \mathrm{E} 858^{13}$, and $\mathrm{E} 886 .^{14}$ The measurements were made in somewhat different $\mathrm{p}_{t}$ regions, but all were near $y_{n n}$. Extrapolating fits to the $\mathrm{p}_{t}$ spectra shows that the measurements all agree within errors. ${ }^{15}$

E859 shows that the rapidity distribution of antiprotons in central $\mathrm{Si}+\mathrm{Au}$ collisions peaks at $y_{n n}$, and by reconstruction of $\bar{\Lambda}$ decays, that approximately $50 \%$ of the observed $\bar{p}$ in $\mathrm{Si}+\mathrm{Au}$ collisions arise from $\bar{\Lambda}$. In p-nucleus collisions, ${ }^{17,16}$ the antiproton rapidity distribution also peaks at $y_{n n}$, and the target dependence of the number of $\bar{p}$ produced is minimal. The E859 data indicat that the antiproton transverse mass $\left(m_{t}^{2}=m^{2}+p_{t}^{2}\right)$ inverse slopes are similar to, though perhaps slightly lower than, those of protons in the same experiment. This disagrees somewhat with the lower statistics E802 result, which had different slopes for $p$ and $\bar{p}$, and larger $\bar{p} \mathrm{~d}$ $\mathrm{N} /$ dy. The E859 results are from a larger data sample, and are within a few standard deviations from E802. The E859 data imply that interpretations based on differing mean field effects ${ }^{5}$ for $p$ and $\bar{p}$ may not be valid.

It is interesting to evaluate the importance of first collision production of antiprotons at the AGS, as subsequent collisions of the projectile nucleons are not far from the $\bar{p}$ production threshold. The number of $\bar{p}$ per first collision was estimated at 5.2 $\times 10^{-6}$ in the E814 spectrometer for p-p $p^{12,17}$ and $2.5-3.2 \times 10^{-6}$ for $\mathrm{Si}+\mathrm{Al}, \mathrm{Cu}$, $\mathrm{Pb}$ collisions. ${ }^{12}$ This implies that annihilation may be important, however the weak target and centrality dependence is not consistent with only first collision production and annihilation. E858 parameterized their $\bar{p}$ production data with

$$
\sigma_{T O T}^{\alpha}=\left(\pi r^{2}\left[A_{P}^{1 / 3}+A_{T}^{1 / 3}-1\right]^{2}\right)^{\alpha}
$$

and found $\alpha=1.3 \pm 0.1 .^{13}$ One may expect that a peripheral process would yield $\alpha$ $=1 / 2$, surface emission of $\bar{p}$ result in $\alpha=1$, and volume emission give $\alpha=3 / 2$. The fit to the data suggests that annihilation of the antiprotons may not be extremely important. This analysis does not exclude, however, a balance of annihilation by additional $\bar{p}$ production.

E802 found that $\bar{p}$ production in p-nucleus collisions is rather similar to p-p collisions and the inverse slopes of the distributions are consistent with first collision production. ${ }^{16}$ Furthermore, no more than $20 \%$ of the $\bar{p}$ need arise from subsequent collisions. E886 found that first collision production can in fact explain ALL the antiproton yield in $\mathrm{Si}+\mathrm{Pt}$ and $\mathrm{Au}+\mathrm{Pt}$ collisions. ${ }^{14}$ This was interpreted as requiring enhanced production mechanisms in light of the expected $\bar{p}$ annihilation, particularly in $\mathrm{Au}+\mathrm{Pt}$. The lack of dependence on the target mass in p-nucleus collisions is not 
consistent with strong annihilation effects, though of course the number of protons at midrapidity is smaller than in Si or Au on heavy targets.

\section{THEORETICAL WISDOM}

Two event generators have been used to successfully reproduce the antiproton production in Si-nucleus collisions at the AGS. The RQMD model, ${ }^{19}$ which includes the formation and decay of color strings, produces a large excess of $\bar{p}$ in heavy systems at the AGS. ${ }^{8,18}$ The excess arises from multistep excitations of the leading baryons, which become more likely to decay into heavy objects. This excess $\bar{p}$ production is counterbalanced in RQMD by strong final state absorption; approximately $2 / 3$ of all produced antibaryons are annihilated in central $\mathrm{Si}+\mathrm{Al}$ collisions at 14.6 $\mathrm{GeV} /$ nucleon. ${ }^{18}$ In contrast, the hadronic cascade model $\mathrm{ARC}^{20}$ produces most antiprotons in first chance nucleon-nucleon collisions. In ARC, approximately $10 \%$ of the $\bar{p}$ in $\mathrm{Si}+\mathrm{Au}$ come from meson-baryon collisions. The smaller number of produced $\bar{p}$ is balanced by a smaller annihilation probability in ARC, though even in ARC nearly $50 \%$ of the $\bar{p}$ in central $\mathrm{Si}+\mathrm{Au}$ are annihilated. ${ }^{10}$ Large annihilation effects in heavy ion collisions were also predicted by Gavin and collaborators. ${ }^{4}$ Both RQMD and ARC describe the E802 data rather well, despite their different assumptions. It is clear that quantitative comparisons of these models to the other data sets are crucial to disentangling these effects.

We can also help sort out the different physics issues and address the importance of $\bar{p}$ annihilation by looking at CERN energies. With $200 \mathrm{GeV} /$ nucleon heavy ion beams, threshold effects are no longer important and first collision analyses are not relevant, removing one variable from the interpretation. As $\bar{p}$ may be formed just as easily in subsequent collisions, the ratio $\bar{p} / \pi^{-}$may help "calibrate" effects of the varying $\sqrt{s}$ of subsequent nucleon-nucleon collisions on $\bar{p}$ production. At CERN, the baryon density $(\mathrm{dN} / \mathrm{dy})$ at midrapidity is considerably lower than at the AGS, so differences in the treatment of the annihilation of $\bar{p}$ should no longer dominate the model results. However, there are still SOME baryons present, so annihilation effects may be investigated and compared to results in the AGS energy regime.

It has also been pointed out that thermal and chemical equilibrium may not be achieved in these collisions. In that case, the abundances of baryons and antibaryons are not controlled by thermodynamic Boltzmann factors, but by the sizes of coherence domains in a low-temperature quark condensate. ${ }^{21}$ In this case, the baryon-antibaryon production may be larger than in an equilibrated system, and so the antibaryon production provides a good probe of nonperturbative QCD.

\section{NA44 EXPERIMENT}

Experiment NA44 at CERN has measured proton and antiproton spectra with high statistics, and compares p-p (approximated by p-Be), p-nucleus, and nucleusnucleus collisions in the same experiment. NA44 is a second generation experiment, with a focussing spectrometer optimized for momentum resolution $\left(\sigma_{p} / p=0.2 \%\right)$ and identification of charged particles at midrapidity. The trigger level particle identification and high rate capability, along with acceptance for a small number of particles per event, yield high quality single particle spectra, even for rare particles such as 


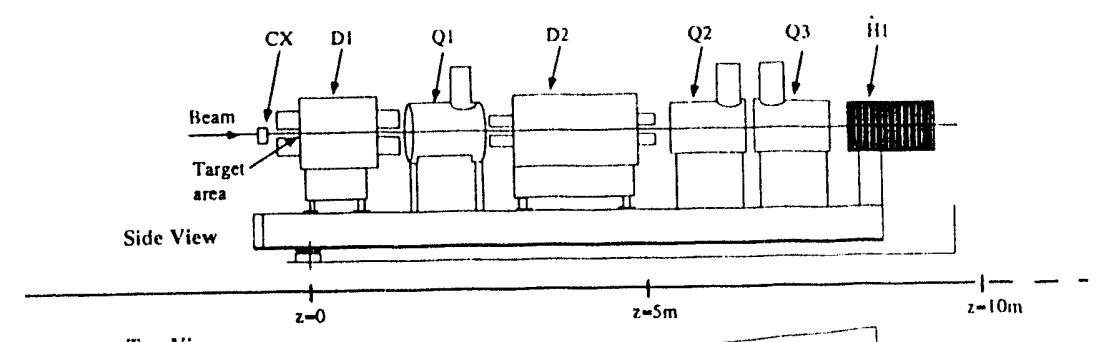

Top View
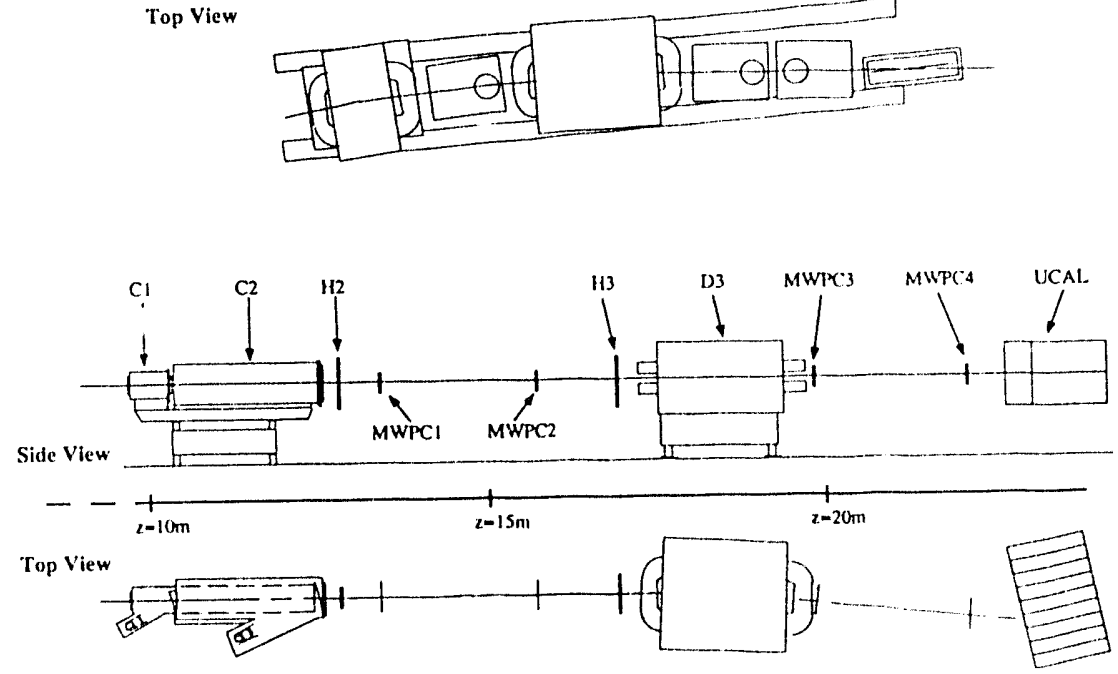

Figure 1: NA44 experiment layout

antiprotons.

The layout of NA44 is shown in Fig. 1. There are three dipole magnets (D1, D2, D3) and three quadrupoles (Q1, Q2, Q3); the first two dipoles select the momentum and the last one is used for momentum calibration. The quadrupoles are configured such that the momentum of the particle is strongly correlated with the position on the first hodoscope. Only one charge state can be detected at one time. The momentum range selected by the spectrometer covers a band of $\pm 20 \%$ around the nominal setting. The angular coverage is approximately -0.3 to 4.5 degrees in the horizontal and \pm 0.3 degrees in the vertical plane. Tracking is done using three highly segmented scintillator hodoscopes ( $\mathrm{H1}, \mathrm{H} 2, \mathrm{H} 3)$, divided into 50, 60, and 50 vertical slats, respectively. Photomultiplier tubes are mounted on either end of the slats to optimize the time-of-flight resolution and allow $y$-position measurement using the time difference between top and bottom.

The beam rate and time-of-flight start are determined with a Cherenkov beam counter (CX) for heavy ion beams, and a forward scintillator (T0) for proton beams. The forward scintillator is used as a triggering device in both cases, to signal either minimum bias or central collisions via the pulse height. A silicon multiplicity detector is used to measure the charged particle distribution with $2 \pi$ acceptance in the pseudorapditiy range $1.5<\eta<3.3$. The minimum bias data in NA44 do contain a bias toward small impact parameters due to the requirement of finding a particle in the spectrometer acceptance. This bias is stronger for more rarcly produced particles. For the sulfur beam, "central" collisions correspond to the top $10 \%$ of the geometric cross section. The charged particle spectra have been corrected for the "target out" contribution.

Particles are identified via time-of-flight measured by the hodoscopes $\left(\sigma_{T O F} \approx\right.$ $100 \mathrm{ps}$ ) and pulse height analysis on the two gas Cherenkov counters. The first 


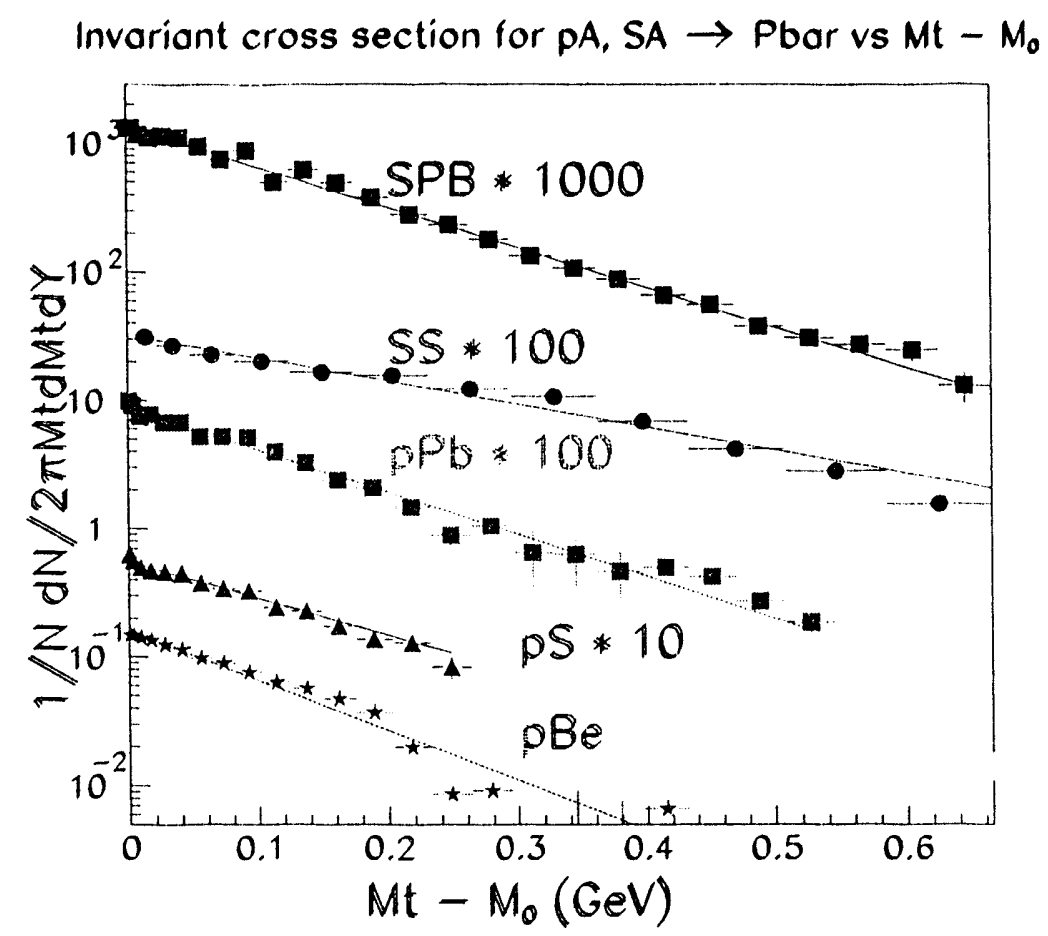

Figure 2: Transverse kinetic energy spectra for antiprotons

Cherenkov (C1) is filled with freon-12 at 1.4 and 2.7 atm. and differentiates between $\pi, \mathrm{K}$ and p. $\mathrm{C} 2$ has a nitrogen/neon mixture at 1.0 and $1.3 \mathrm{~atm}$., and rejects electrons and pions. The contamination of any particle sample by particles of another type is less than $4 \%$.

The spectrometer single particle acceptance for protons and antiprotons consists of a rapidity slice of 0.3 units, centered about $y=2.8$. The $p_{t}$ range is from $0-800$ $\mathrm{MeV} / \mathrm{c}$. A second spectrometer setting provides $\mathrm{p}$ and $\bar{p}$ distributions centered about $y=2.0$.

Tracks are reconstructed from the recorded positions on the three hodoscopes, with pattern recognition constrained by straight-line trajectories after the magnets. The cross sections have been corrected for geometrical acceptance, multiple scattering, particle identification cuts, and pattern recognition losses using a Monte Carlo simulation of the spectrometer. The simulated events are reconstructed using the full analysis chain, and the input distribution is tuned to the observed particle spectra. The uncertainty on the $p_{t}$ measurement is $0.15 \%$, arising from multiple scattering and the hodoscope granularity. The systematic uncertainty on the $p_{t}$ scale is $1.6 \%$.

\section{TRANSVERSE MASS DISTRIBUTIONS}

Particle distributions are often shown as a function of $m_{t}$, the transverse mass, or $k_{t}$, the transverse kinetic energy $\left(k_{t}=m_{t}-m_{0}\right)$. This is motivated by the possibility of describing the observed distributions with a thermal model; an exponential distribution in $m_{t}$, common to all particle species, might be expected for thermal emission. ${ }^{22}$ Figure 2 shows the NA44 transverse kinetic energy distributions ${ }^{23}$ of antiprotons from $200 \mathrm{GeV} /$ nucleon $\mathrm{S}+\mathrm{Pb}, \mathrm{S}+\mathrm{S}$, and $450 \mathrm{GeV} \mathrm{p}+\mathrm{Pb}, \mathrm{S}$ and $\mathrm{Be}$. The lines are the result of fits with an exponential $k_{t}$ distribution.

The NA35 collaboration has investigated the decay of $\bar{\Lambda}$, and the contribution of 


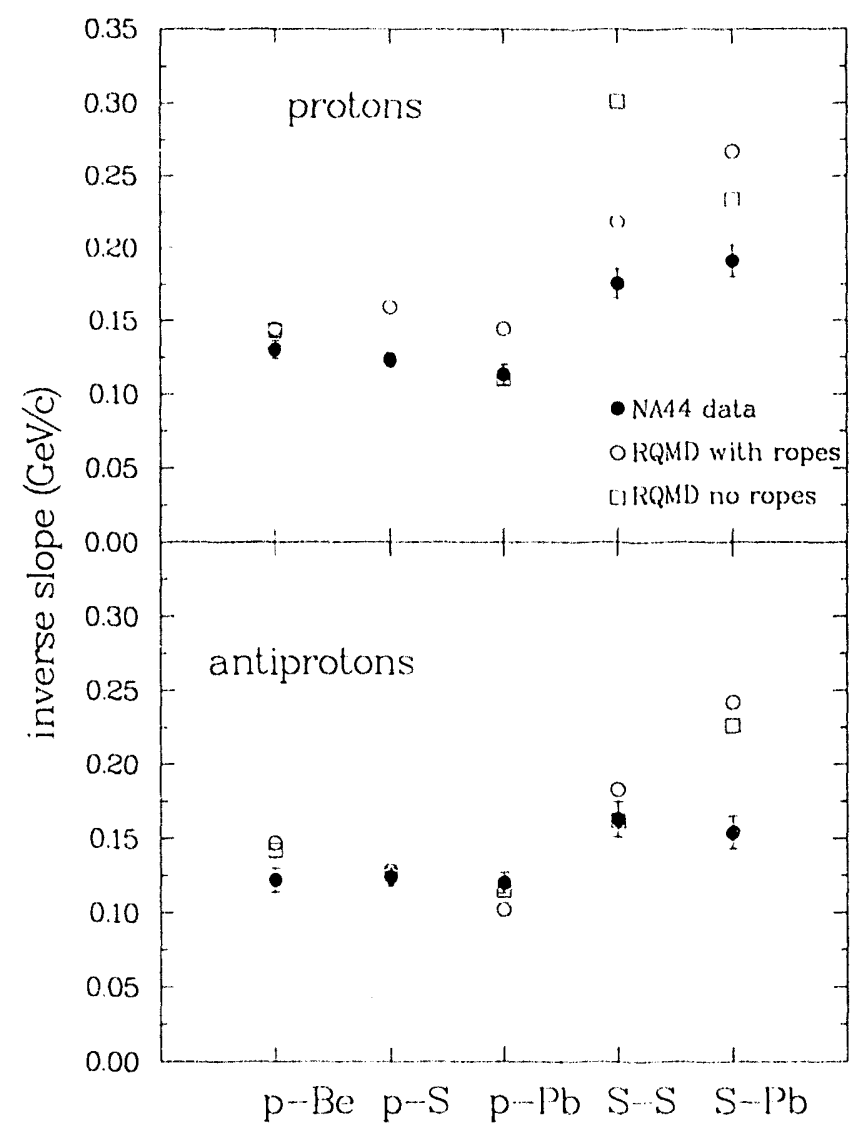

Figure 3: Inverse slopes of proton and antiproton spectra

these decays to the measured $\bar{p} .24,25$ They find that approximately $30 \%$ of $\bar{p}$ in their acceptance come from $\bar{\Lambda}$ decay. We have used RQMD events and our spectrometer acceptance to investigate the effect of $\bar{\Lambda}$ decay upon our antiproton spectra, and find that we reconstruct all $\bar{p}$ arising from $\bar{\Lambda}$. The $\bar{\Lambda}$ contribution is $30 \%$ in our data as well, but does not significantly affect shape of the antiproton $k_{t}$ distribution.

The inverse slopes resulting from the fits in Fig. 2 are shown in Fig. 3 along with the inverse slopes for protons, plotted as a function of the system size. It is clear that both protons and antiprotons have flatter spectra, and larger inverse slopes, in nucleus-nucleus than in p-nucleus collsions. The system size dependence of the $p$ and $\bar{p}$ spectra is considerably stronger than for pions (which show almost no system dependence) or kaons. ${ }^{22}$ In $\mathrm{S}-\mathrm{S}$ and $\mathrm{S}-\mathrm{Pb}$ collisions, the $\bar{p}$ slope is $1-2$ standard deviations lower than the proton slope. NA35 reported at this school on protons and antiprotons identified in the TPC; their $\bar{p}$ inverse slopes agree with NA44 at a similar level. We are currently analyzing additional heavy ion data to reduce the statistical errors on these slopes in urder to definitively determine the magnitude of the difference.

Pig. 3 also shows the inverse slopes of protons and antiprotons at midrapidity from the RQMD event generator, as a function of the size of the system. The inverse slopes were determined by fitting an exponential as in the data. Events were generated using RQMD in two modes - with and without the fusion of color strings into color ropes. As expected, the results are the same for p-nucleus collisions which have few nucleonnucleon collisions and correspondingly few strings which may fuse. In heavy ion 


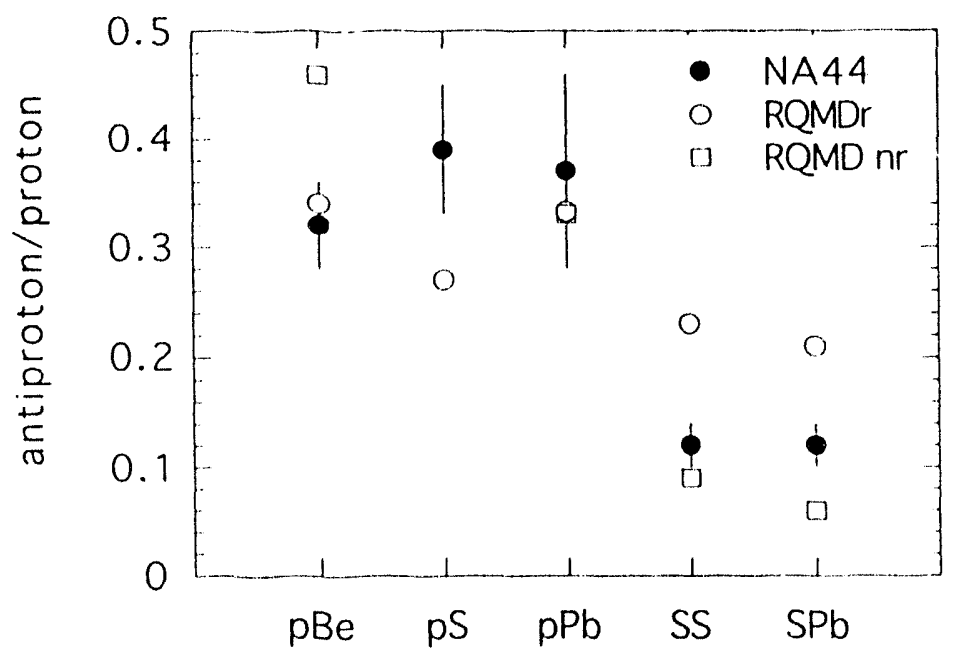

Figure 4: ratio of $\bar{p} / \mathrm{p}$ for different systems

collisions, the probability of string fusion is considerably larger, ${ }^{9}$ but the effect on the particle momenta is small. Both sets of RQMD calculations successfully reproduce the trends of the slopes with system size. RQMD appears to somewhat overpredict the slopes in $\mathrm{S}+\mathrm{Pb}$ collsions, but the difference is not very large. In RQMD, the difference between proton and antiproton spectra is small.

\section{PROTON AND ANTIPROTON YIELD}

The spectra shown in Fig. 2 were integrated to determine the yield of antiprotons in these collisions; proton distributions were similarly analyzed. The fits were extrapolated to high $p_{t}$, beyond the region of measurement, to allow calculation of the rapidity densities. Fig. 4 shows the ratio of $\mathrm{dN} / \mathrm{dy}$ for $\vec{p}$ and $p$ for the various target-projectile systems. The $p+B e$ ratio agrees reasonably well with the value observed at the ISR. ${ }^{2}$ It is rather remarkable that the ratio remains approximately constant for protons on various nuclear targets, then decreases in nucleus-nucleus collisions. Of course, the $\bar{p} / p$ ratio is affected by the proton distributions as well as antiproton production. It is well known that collisions of heavier systems result in a larger number of protons at midrapidity. ${ }^{6,7}$ Even proton collisions on heavy targets result in a shift of target protons to larger rapidity. ${ }^{7}$ These trends alone may be able to explain the system size dependence observed, though we must also investigate the possible effects of excess $\bar{p}$ production and $\bar{p}$ annihilation. The latter will certainly depend sensitively on $\mathrm{p} d \mathrm{~N} / \mathrm{dy}$, magnifying the effect of midrapidity protons on this ratio.

A powerful tool to address these questions is an event generator containing $\bar{p}$ annihilation at a known level. RQMD provides this feature and can produce excess $\bar{p}$ if string fusion to color ropes is included. ${ }^{9}$ The $\bar{p} / p$ ratios from RQMD with and without string fusion for the various systems are compared with the data in Fig. 4. Both sets of RQMD calculations reproduce the trends observed in the data, though $\bar{p} / p$ in the RQMD version without string fusion falls somewhat more steeply. Such behavior may be expected if produced antiprotons are annihilated, and this loss is not balanced by increased production. 


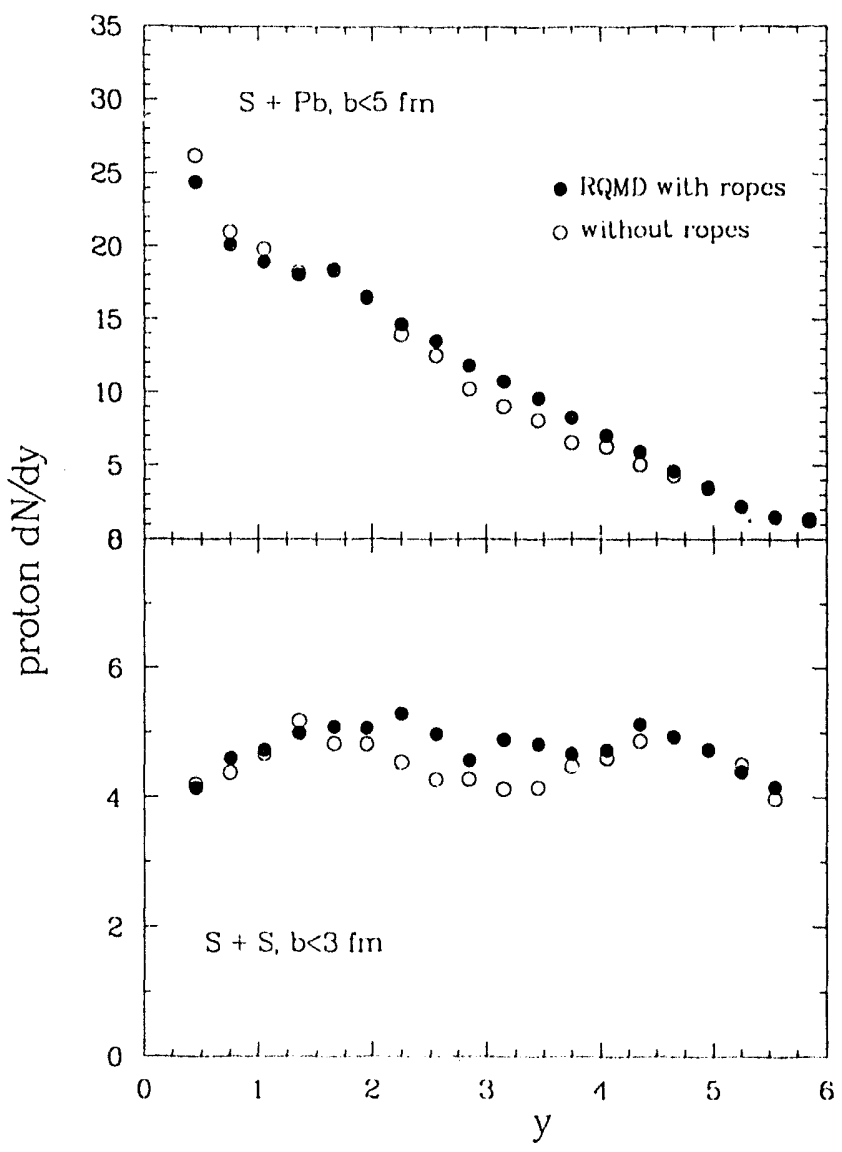

Figure 5: Rapidity distribution of protons from RQMD with and without string fusion to color ropes

\section{THE MODEL VIEW OF ANTIPROTON PRODUCTION AND ANNI- HILATION}

Fig. 5 shows the proton rapidity distributions predicted by RQMD for $\mathrm{S}+\mathrm{Pb}$ and $\mathrm{S}+\mathrm{S}$ collisions. The impact parameter range was chosen to match the NA44 trigger; the requirement of large pulseheight in the T0 scintillator was simulated using RQMD events as input and including the geometry and response of the trigger scintillator. 'The closed points are from RQMD events including string fusion to color ropes, and the open points without rope formation. This comparison shows that string fusion does not affect the proton distributions. This may be expected as the proton yield is dominated by protons already existing in the projectile and target, rather than by protons produced in the collision. The predictions in Fig. 5 can be compared with proton production data from $\mathrm{HELIOS}^{7}$ and with preliminary NA44 results. ${ }^{23}$ HELIOS found proton $\mathrm{dN} / \mathrm{d} y=18 \pm 0.9$ at $y=1$ in central $\mathrm{S}+\mathrm{W}$ collisions. NA44 sees $16 \pm 4$ protons at $y=2.1$ and $14 \pm 4$ at $y=2.8$. Clearly, the RQMD model successfully reproduces the proton rapidity distribution.

$\Lambda$ s the proton yields are correct in the model, we may use it to study the competing effects on antiproton production in these systerns. The RQMD predictions for the $\bar{p}$ rapidity distributions are shown in Fig. 6. Urlike for protons, the antiproton yield is strongly enhanced (by factors of 2-3) by formation of color ropes. This entancement is similar to that observed in RQMD for $\bar{\Lambda}$ production, ${ }^{9}$ which agrees with data ${ }^{24}$ and implies that enhanced antibaryon production signals more collective behavior in 


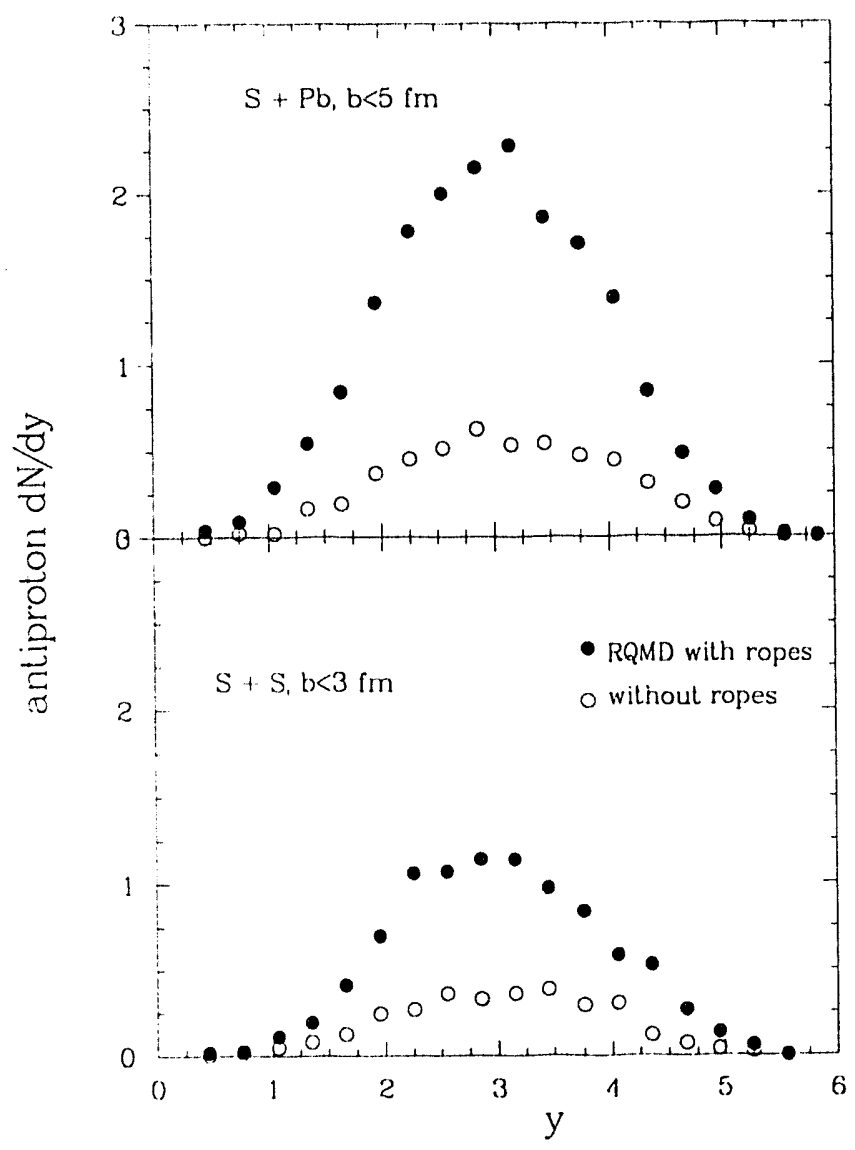

Figure 6: Rapidity distribution of antiprotons from RQMD with and without string fusion to color ropes

heavy ion collisions than just superposition of $\mathrm{p}-\mathrm{p}$ collisions. ${ }^{9,25}$. Preliminary results from NA44 on $\bar{p} \mathrm{dN} / \mathrm{d} y$ at $y=2.8$ are $1.7 \pm 0.6$ for $\mathrm{S}+\mathrm{Pb}$ and $0.8 \pm 0.2$ for $\mathrm{S}+$ $\mathrm{S}$ collisions. NA35 has reported very similar preliminary values at this conference for antiprotons identified in the TPC. ${ }^{25}$ These $\bar{p}$ yields are considerably closer to the predictions of RQMD with color rope formation than without, and support the conclusions drawn from the $\bar{\Lambda}$ results. Of course, the $\bar{p}$ measurement is not totally independent of the $\bar{\Lambda}$ yield, as $30 \%$ of the $\bar{p}$ arise from $\bar{\Lambda}$ decays. However, this contribution alone does not remove the need to include color ropes in the model to reproduce the observed $\bar{p}$ yields.

As described above, the RQMD model has a large cross section for annihilation of $\bar{p}$ with protons. At AGS energies, the baryon density at midrapidity is large, ${ }^{26}$ and a large fraction of the secondary collisions experienced by produced particles are with baryons. The annihilation of $\bar{p}$ is the dominant process determining the number of $\bar{p}$ produced in the model at those energies. At CERN, the ratio of $\pi / p$ at midrapidity is much larger, ${ }^{23,6}$ and most secondary collisions are with mesons. In this case, we may expect less $\bar{p}$ annihilation and a greater sensitivity to the other processes affecting the antiproton yield.

We try to quantify the annihilation effects at CERN by using RQMD and counting the number of collisions suffered by each proton and antiproton before exiting the system. These are plotted as a function of the final rapidity of the $\mathrm{p}$ and $\bar{p}$ for $\mathrm{S}+\mathrm{Pb}, \mathrm{S}+\mathrm{S}$ and $\mathrm{p}+\mathrm{Be}$ in Fig. 7. The events used for this analysis come from 


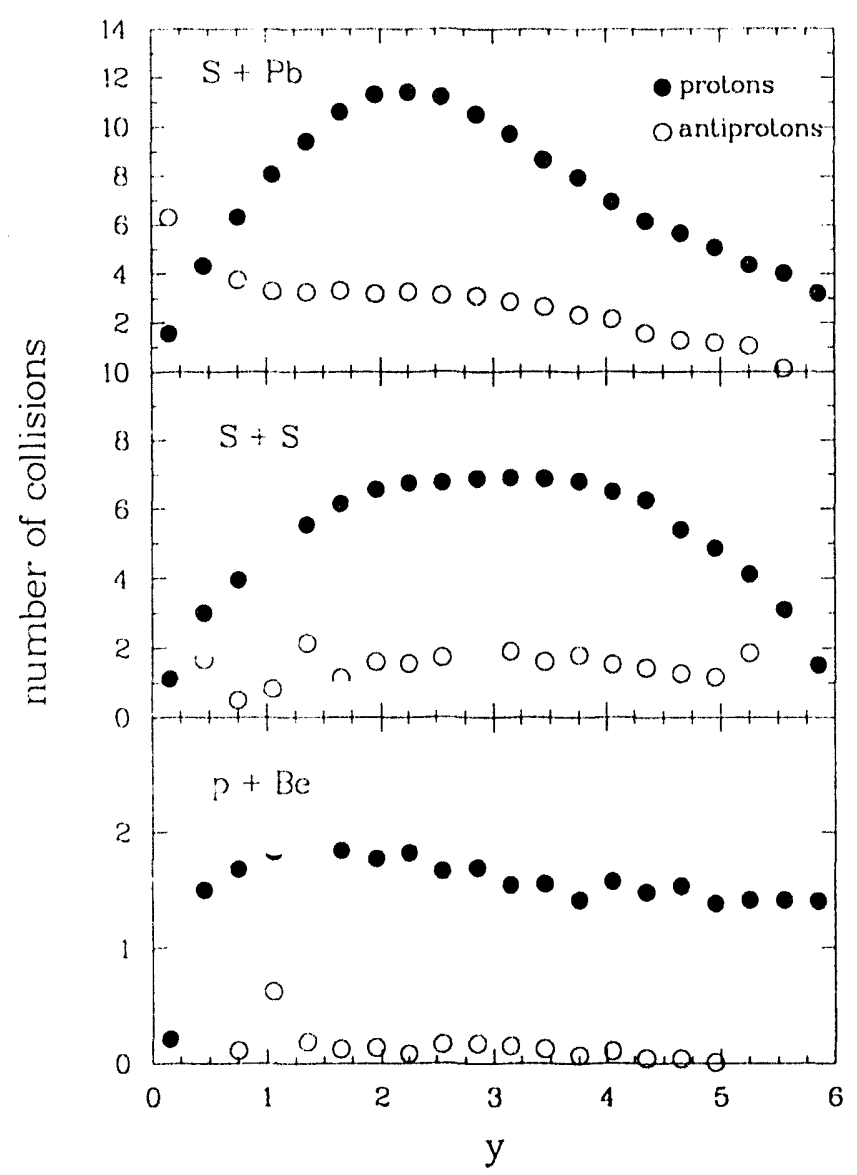

Figure 7: Number of collisions (primary + secondary) of $\mathrm{p}$ and $\bar{p}$ in RQMD, as a function of rapidity. $\mathrm{S}+\mathrm{S}$ and $\mathrm{S}+\mathrm{Pb}$ are central collisions.

very central collisions ( $b \leq 1 \mathrm{fm}$ ) and include string fusion. It is immediately obvious that protons undergo many more collisions than antiprotons in all of the systems. However, the collision count includes PRIMARY collisions of the particles as well as secondary collisions, accounting for at least some of this difference. This may be accounted for by recalling that simple geometrical models which superimpose $\mathrm{p}+\mathrm{p}$ collisions at differing $\sqrt{s}$ can successfully reproduce the number of pions formed in both $\mathrm{p}+\mathrm{A}$ and $\mathrm{A}+\mathrm{A}$, and consequently do a good job in counting the number of primary nucleon-nucleon collisions. ${ }^{27}$ Using such a geometrical picture for central collisions, we estimate that prot ns undergo an average of 1.5 collisions in $\mathrm{p}+\mathrm{Be}$ and 2.5 - 3 primary collisions in $\mathrm{S}+\mathrm{S}$. In $\mathrm{S}+\mathrm{Pb}$, projectile protons suffer approximately 6 primary collisions and target protons about 4 . Subtracting these numbers from the collisions experienced by midrapidity protons shown in Fig. 7 shows that there remains a difference in the number of secondary collisions of protons and antiprotons in nucleus-nucleus at CERN. Antiprotons experience an average of 2 - 3 fewer secondary collisions than protons in both $\mathrm{S}+\mathrm{S}$ and $\mathrm{S}+\mathrm{Pb}$. This implies that annihilation of antiprotons does occur at CERN energies.

We have attempted to study the importance of $\bar{p}$ annihilation from the NA44 data by looking at the system dependence of the $\mathrm{p} / \pi^{+}$and $\bar{p} / \pi^{-}$ratios. Such an approach uses the pion yields to "count" the number of nucleon-nucleon collisiors and normalize for the varying $\sqrt{s}$ of these collisions. This kind of analysis is qualitative rather than quantitative, but may be used to check if the data are consistent with the conclusions 
drawn from the model; the actual values of the ratios are strongly affected by the spectrometer acceptance and fact that we measure protons around $y=2.8$ and pions at $y=3.5-4.5$. Nevertheless, the trends are informative. In p-nucleus collisions (which used $450 \mathrm{GeV}$ proton beam), the $\mathrm{p} / \pi^{+}$and $\bar{p} / \pi^{-}$ratios do not depend on the target size. $\mathrm{p} / \pi^{+}$exceeds $\bar{p} / \pi^{-}$, as we would naively expect. In $\mathrm{S}+\mathrm{Pb}$ and $\mathrm{S}$ $+\mathrm{S}, \mathrm{p} / \pi^{+}$is larger than in p-nucleus collisions, consistent with the shift of protons toward midrapidity shown in Fig. 5 and supported by data. ${ }^{6,7}$ To compare $\bar{p} / \pi^{-}$in nucleus-nucleus and p-nucleus collisions, we must first take into account the fact that the $\bar{p}$ production cross section falls by $\approx 25 \%$ from $450 \mathrm{GeV}$ to $200 \mathrm{GeV}$ beams, but the $\pi$ cross section falls by only $\approx 10 \%$. The observed $\bar{p} / \pi^{-}$ratio in $\mathrm{S}+\mathrm{Pb}$ and $\mathrm{S}+\mathrm{S}$, however, is lower than p-nucleus by more than this trivial drop. Such an additional reduction in $\bar{p} / \pi^{-}$can be expected if antiprotons are annihilated. The FRITIC $F$ model, ${ }^{28}$ which includes no $\bar{p}$ annihilation or excess production, reproduces the $\mathrm{p}$ nucleus $\bar{p} / \pi^{-}$ratio adequately but predicts no change upon going to nucleus-nucleus collisions; it fails miserably on the proton ratios and rapidity distributions, as is well known. ${ }^{7}$ RQMD reproduces the general trend in the ratios observed in NA44.

\section{ANTIDEUTERON PRODUCTION}

NA44 can also look for antideuterons, using a unique combination of tools for $\bar{d}$ identification and background rejection. The focussing spectrometer gives excellent momentum resolution, which coupled with the very good time-of-flight resolution allows a precise measurement of particle masses. The Cherenkov rejection of pions and kaons greatly reduces the background and makes the main identification problem $p$-d separation. We also use the uranium/scintillator calorimeter at the end of the NA44 spectrometer to reject kaon and pion decay background and require the $\bar{p}$ or $\bar{d}$ annihilation energy. The combination of these cuts results in rather clean mass spectra; positive tracks show a clear deuteron peak.

Though the statistics of $\bar{d}$ are too small to show a peak, a preliminary analysis shows $4 \bar{d}$ candidates in $\mathrm{S}+\mathrm{Pb}$ collisions. From the deuterons observed in the same size sample, we may estimate the number of antideuterons expected using the coalescence model. ${ }^{29}$ This yields a prediction of about $4 \bar{d}$ in our sample. We are currently analyzing a larger data sample to extract a more statistically significant result, but it appears that antideuteron production at CERN energy is range expected from coalescence of antinucleons. This differs from AGS energies, where the $\bar{d}$ cross section is lower than predicted by coalescence by a factor of $10 . .^{13}$ This difference may serve to again underscore the lesser importance of antinucleon annihilation at CERN than at AGS energy.

\section{CONCLUSIONS}

We have discussed the complex tradeoff between threshold effects on the production cross section of antiprotons, and the amount of annihilation in nucleus-nucleus collisions at AGS energies. We use measurements at CERN, where threshold effects are not important, to gain further insight. We find that there is excess antiproton production which cannot be explained by models incorporating no collective behavior of the nucleon-nucleon collisions. The RQMD model including fusion of strings into 
color ropes agrees well with the observed antiproton yields in $\mathrm{S}+\mathrm{Pb}$ and $\mathrm{S}+\mathrm{S}$ collisions. The $30 \%$ contribution of $\bar{\Lambda}$ decays to the $\bar{p}$ yield is not sufficient to account for the enhanced $\bar{p}$ production; according to RQMD, $\bar{\Lambda}$ and $\bar{p}$ enhancement are both due to the same mechanism anyway. It appears that some antiproton annihilation occurs at CERN energy, but is less important than at the AGS. The proton and antiproton $m_{t}$ inverse slopes are fairly similar and do not leave room for large mean field effects on the $\bar{p}$ distibutions.

The NA44 collaboration is currently analyzing a larger data set, extracting antideuteron yields, and working on improving the systematic errors. This along with $\mathrm{Pb}+\mathrm{Pb}$ results from CERN, and $\mathrm{Au}+\mathrm{Au}$ at the AGS will help constrain the models and fix production and annihilation cross section Armed with these, we may better quantify the magnitude of collective effects on heavy particle production in nucleus-nucleus collisions.

\section{ACKNOWLEGEMENTS}

I would like to thank Heinz Sorge, Michael Murray, Jehanne Simon-Gillo, John Sullivan, and Doug Fields for many enlightening discussions about both data and the RQMD model results. Their invaluable aid in compiling and analyzing the model runs for the various systems is most appreciated. This work was supported by the U.S. Department of Energy.

\section{REFERENCES}

1. W. Bell, et al., Z. Phys. C27, 191 (1985).

2. B. Alper, et al., Nucl. Phys. B100, 237 (1975).

3. U. Heinz, P.R. Subramanian and W. Greiner, Z. Phys. A318, 247 (1984); P. Koch, B. Mueller, H. Stoecker and W. Greiner, Mod. Phys. Lett. A3, 737 (1988).

4. S. Gavin, M. Gyulassy, M. Pluemer and R. Venugopalan, Phys. Lett. B234, 175 (1990).

5. V. Koch, G.E. Brown and C.M. Ko, Phys. Lett. B265, 29 (1991).

6. H. Stroebele, et al. (NA35 Collaboration), Nucl. Phys. A525, 59c (1991).

7. T. Akesson, et al. (HELIOS Collaboration), Z. Phys. C53, 183 (1992).

8. A. Jahns, H. Sorge, H. Stoecker and W. Greiner, Phys. Rev. Lett. 68, 2895 (1992).

9. H. Sorge, M. Berenguer, H. Stoecker and W. Greiner, Phys. Lett. B289. 6 (1992).

10. S.H. Kahana, Y. Pang, T. Schlagel and C.B. Dover, Phys. Rev. C.47, R1356 (1993).

11. T. Abbott, et al. (E802 collaboration), Phys. Lett. B271, 447 (1991) and Quark Matter '93. 
12. J. Barette, et al. (E814 collaboration), Phys. Rev. Lett. 70, 1763 (1993).

13. A. Aoki, et al. (E858 collaboration), Phys. Rev. Lett. 69, 2345 (1992).

14. G. Diebold, et al. (E886 Collaboration), Phys. Rev. C48, 2984 (1993).

15. B. Shiva Kumar, Proc. of the XXVI International Conference on High Energy Physics, Dallas TX, August 1992, p. 1006; B. Shiva Kumar, Proc. of HIPAGS '93, Boston MA, Jan. 1993.

16. T. Abbott, et al. (E802 Collaboration), Phys. Rev. C47, R1351 (1993).

17. J.V. Allaby, et al., CERN Report No. 70-12, 1970 (unpublished).

18. A. Jahns, H. Sorge, H. Stoecker and W. Greiner, Z. Phys. A341, 243 (1992); A. Jahns, C. Spieles, R. Mattiello, H. Stoecker, W. Greiner and H. Sorge, Phys. Lett. B308, 11 (1993).

19. H. Sorge, H. Stoecker and W. Greiner, Ann. Phys. 192, 266 (1989).

20. Y. Pang, T.J. Schlagel and S.H. Kahana, Phys. Rev. Lett. 68, 2743 (1992).

21. J. Ellis, U. Heinz and H. Kowalski, Phys. Lett. B233, 223 (1989).

22. B. V. Jacak, Proc. of the NATO School on Particle Production in Highly Excited Matter, Il Ciocco, Italy, July 1992, p.471.

23. M. J. Murray, Proc. of Quark Matter '93, Borlange, Sweden, June 1993.

24. J. Bartke, et al. (NA35 Collaboration), Z. Phys. C48, 191 (1990).

25. D. Roerich (NA35 Collaboration), these proceedings.

26. T. Abbott, et al. (E802 Coilaboration), Phys. Rev. Lett. 66, 1567 (1991).

27. A. D. Jackson and H. Boggild, Nucl. Phys. A470, 660 (1987).

28. B. Andersson, G. Gustafson, and B. Nilsson-Almquist, Nucl. Phys. B281, 289 (1977).

29. H.H Gutbrod, et al., Phys. Rev. Lett. 37, 667 (1976); C. B. Dover, U. Heinz, E. Schnedermann, and J. Zimanyi, Phys. Rev. C44, 1636 (1991). 



$$
\longrightarrow
$$
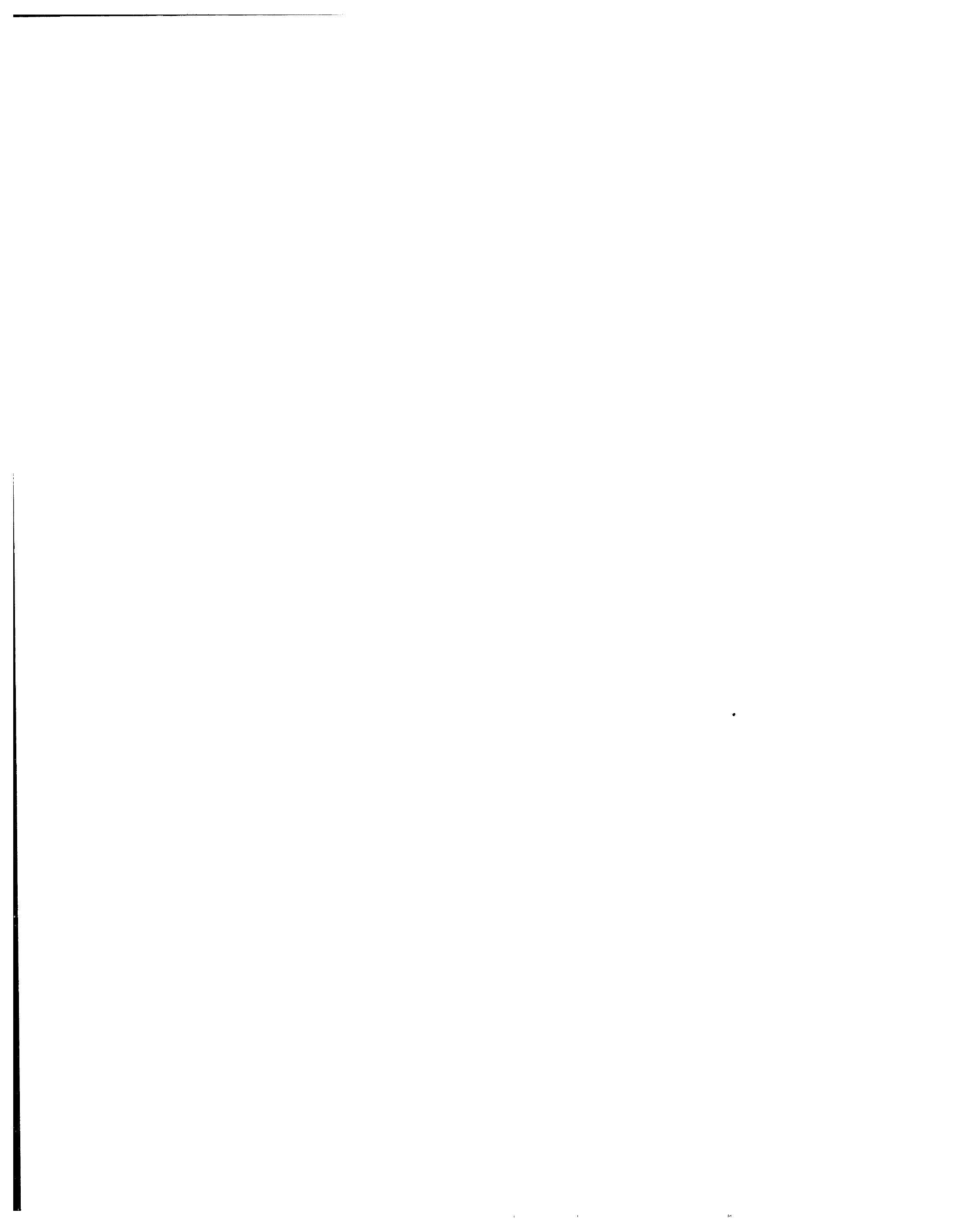

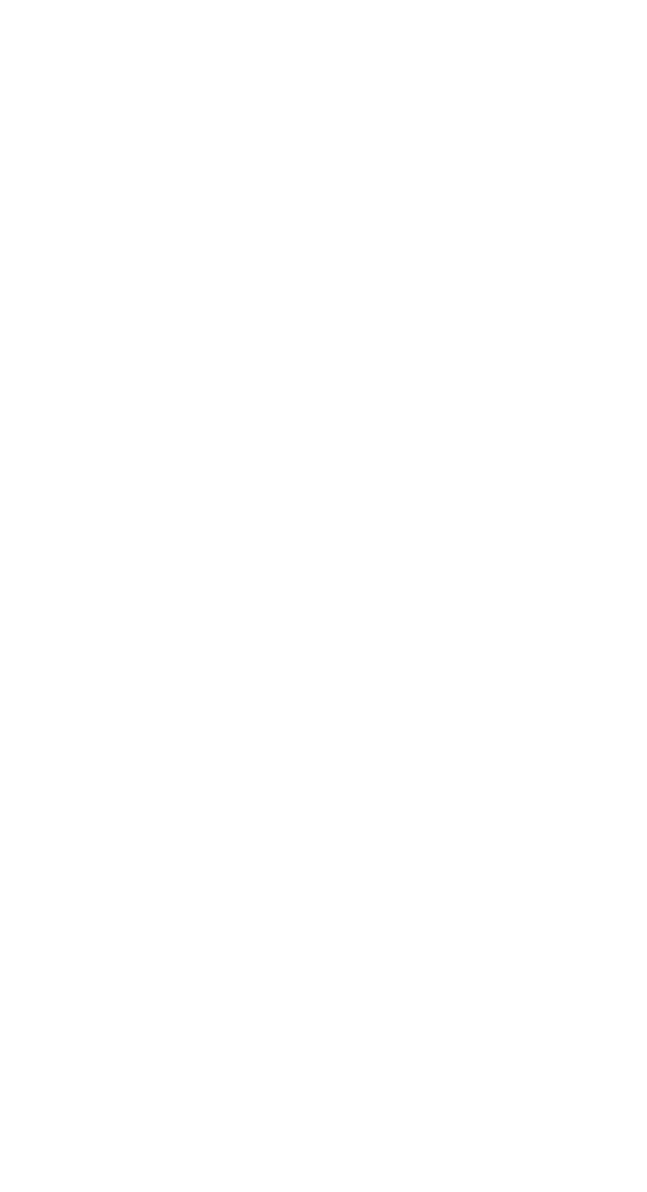

